\title{
PROJETO DO PERFIL AERODINÂMICO PARA O DESENVOLVIMENTO DE UMA AERONAVE RÁDIO CONTROLADA DA EQUIPE TUIUIU AERODESIGN DA UCDB
}

\author{
Willian Pires Sabadini Zuquil) (wpszuqui@gmail.com), Fabiano Pagliosa Branco ${ }^{(1)}$. (fabiano@ucdb.br)
}

(1) Universidade Católica Dom Bosco (UCDB); Departamento de Engenharia Mecânica

RESUMO: Com a finalidade de incentivar os acadêmicos das engenharias nas ciências aeronáuticas, a Sociedade dos Engenheiros Automotivos (SAE) criou a competição SAE Aerodesign que desafia diversas equipes, formada por acadêmicos das engenharias. A Universidade Católica Dom Bosco compete com a equipe Tuiuiú Aerodesign que participará do torneio de acesso de 2020. Dessa forma, esse trabalho propõe o projeto do perfil aerodinâmico da aeronave radio controlada, onde buscou-se compreender os efeitos das características geométricas do perfil aerodinâmico, principalmente a espessura e arqueamento, e como impactam na aerodinâmica e no desempenho da aeronave, utilizando-se o software XFLR5. Com base nos resultados das análises, criou-se um perfil, denominado 12412, e comparou-se com o perfil Eppler 423, comumente usados pelas equipes nesse tipo de competição. O novo perfil apresentou melhor desempenho na velocidade de estol e distâncias necessárias para pouso e decolagem, entretanto com uma redução na tração requerida que impacta diretamente na escolha do motor.

PALAVRAS-CHAVE: AEROFÓLIO, PROJETO, ENGENHARIA AERONÁUTICA, SAE AERODESIGN.

\section{AERODYNAMIC AIRFOIL PROJECT FOR THE DEVELOPMENT OF A CONTROLLED RADIO AIRCRAFT}

ABSTRACT: In order to encourage scholars of engineering in the aeronautical sciences, the Society of Automative Engineers (SAE) created the SAE Aerodesign competition that challenges several teams, formed by engineering academics. The Universidade Católica Dom Bosco (UCDB) competes with the Tuiuiú Aerodesign team that will participate in the Access tournament in 2020. In this way, this work proposes the airfoil design of the radio controlled aircraft, where it was sought to understand the effects of the geometric characteristics of the airfoil, especially the thickness and arching, and how they impact on the aerodynamic and aircraft performance, using the software XFLR5. Based on the results of the analyses, an airfoil, called 12412, was created and compared with the Eppler 423 airfoil, commonly used by teams in this kind of competition. The new airfoil presented better performance in the speed of stall and distances needed for landing and takeoff, however with a reduction in the required traction that directly impacts on the choice of the engine.

KEYWORDS: AIRFOIL, DESIGN, AERONAUTICAL ENGINEERING, SAE AERODESIGN. 


\section{$\mathbf{X I X}$ \\ CENEMI}

\section{INTRODUÇÃO}

Com a finalidade de incentivar os acadêmicos das engenharias na ciência aeronáutica, a Sociedade dos Engenheiros Automotivos (SAE) criou a competição SAE Aerodesign que desafia diversas equipes, formada por acadêmicos das engenharias. O objetivo é a construção de uma aeronave rádio controlada com limitações de projeto impostas pelo comitê da competição para aumentar a criatividade dos alunos, tornando-os hábeis a resolver problemas que muitos engenheiros no ramo da aeronáutica se deparam em seu dia a dia (portal.saebrasil.org.br/programas-estudantis/sae-brasil-aerodesign).

Em 2012 os acadêmicos da Universidade Católica Dom Bosco criaram a Equipe Tuiuiú, sua primeira participação foi no mesmo ano de sua criação, e o primeiro aeromodelo construído pela equipe ficou na posição 63 de 68 participantes. Em 2019, a equipe Tuiuiú Aerodesign participará do torneio de acesso para a competição de 2020 com um novo aeromodelo, e para tal um projeto aerodinâmico bem fundamentando é exigido, com escolha do perfil aerodinâmico adequado com análises computacionais.

Segundo Mathias (2015), desde a época dos irmãos Wright, quando a insistência deles em tentar levantar voo numa pequena aeronave levou a diversas experiências em túnel de vento rudimentares para estudar os efeitos aerodinâmicos em perfis, busca-se melhorar e entender os efeitos do escoamento do ar em perfis aerodinâmicos, pois conforme Raymer (1992) o perfil, em muitos aspectos, é o coração do avião, já que afeta a qualidade da asa e o desempenho da aeronave em pouso e decolagem, bem como seu controle em voo.

Para atender o objetivo da competição SAE Aerodesign que tem como característica principal o desenvolvimento de uma aeromodelo cargueiro com velocidade menor que $30 \mathrm{~m} / \mathrm{s}$ e altitudes abaixo de 1500 m, os perfis devem apresentar um alto valor de coeficiente de sustentação aliado a baixos valores de coeficientes de arrasto e momento, resultando em uma elevada eficiência aerodinâmica, conforme Rodrigues (2011). Os principais modelos de aerofólios são o Eppler 423, Selig 1210 e Wortmann FX 74-CL5-140.

As análises dos perfis são realizadas através de cálculos matemáticos complexos e há diversas hipóteses físicas utilizadas. Para facilitar e acelerar o procedimento, utiliza-se softwares que simulam o escoamento de fluidos em corpos, conhecidos como Computational Fluid Dynamics (CFD) ou que simulam um túnel de vento. Dentre vários presentes no mercado, destaca-se o XFLR5 porque é gratuito, resolução rápida, permite analisar múltiplos perfis simultaneamente e fornece múltiplos gráficos para a análise.

Dessa forma, o objetivo desse trabalho foi compreender o efeito da espessura e arqueamento (ou camber) nos coeficientes de sustentação e arrasto, através das análises dos perfis NACAs 4 dígitos 


\section{XIX \\ CENEMI}

no software XFLR5. Posteriormente, com a base de um perfil NACA 4 dígitos, criou-se um perfil específico para o projeto do aeromodelo e comparou-se com o aerofólio Eppler 423, de maneira que com o conhecimento dos efeitos das características geométricas buscou-se incentivar a criação e desenvolvimento de novos perfis aerodinâmicos para a competição SAE Aerodesign.

\section{MÉTODOS}

As análises dos perfis estudados foram realizadas no software XFLR5, em todas adotou-se um número de Reynolds de 500.000, devido as baixas velocidades do aeromodelo onde os valores ficam entre 300.000 a 500.000 segundo Rodrigues (2011). Simulando-se um túnel de vento, é necessário saber o nível de turbulência do meio onde o perfil se encontraria, e esse nível de turbulência é dado pela variável "Ncrit" do software, sendo esse valor variando de 4 a 14, onde quanto menor o seu valor maior é a turbulência do túnel de vento simulado. Adotou-se um valor padrão para "Ncrit" de valor igual a 9, onde representaria um túnel de vento médio com um controle do escoamento bom onde permita-se um escoamento laminar (www.web.mit.edu/drela/Public/web/xfoil/xfoil_doc.txt). Como o número de Reynolds relaciona velocidade e massa específica, que por sua vez é influenciada pela altitude de voo do aeromodelo, não é necessário adotar um valor para o número de Mach (www.web.mit.edu/drela/Public/web/xfoil/xfoil_doc.txt). Por fim, o escoamento viscoso foi adotado nas análises.

Como um escoamento do tipo laminar depende de uma superfície lisa e sem perturbações, e essa condição é difícil de se obter em voo, adotou-se uma transição forçada de 0,35 no extradorso e no intradorso do perfil, sendo a transição forçada um ponto do perfil onde o escoamento passa de laminar para turbulento, e esse ponto da transição forçada varia em seu valor de 0 a 1 , sendo o valor 0 considerado o início do perfil (bordo de ataque) e o valor 1 o final do perfil (bordo de fuga). A Figura 1 destaca as principais características geométricas de um perfil aerodinâmico.

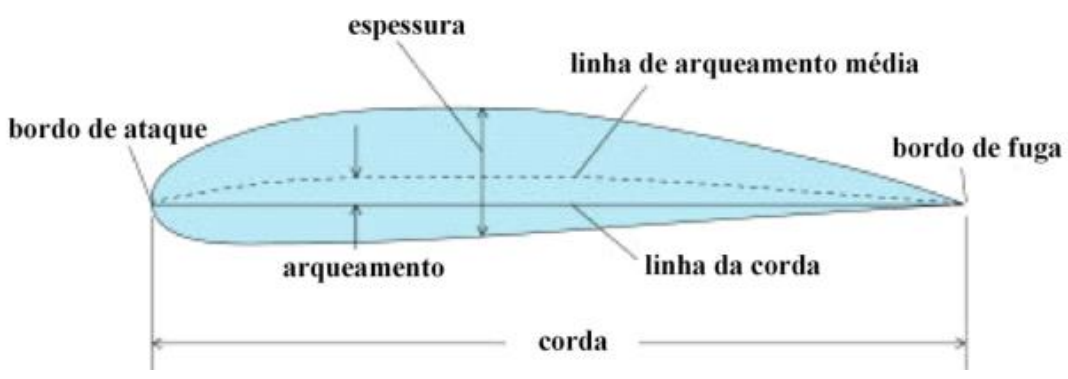

FIGURA 1. Características geométricas de um perfil aerodinâmico. Fonte: RODRIGUES (2011). 


\section{$\mathrm{XIX}$ \\ CENEMI}

Na Figura 1 a corda (c) é o comprimento do perfil ou linha da corda, que liga o bordo de ataque (frente do perfil) ao bordo de fuga (traseira do perfil). A espessura (t), dada em porcentagem de corda (t/c), é a distância do extradorso ao intradorso, sendo o valor máximo o adotado nas nomenclaturas dos perfis. O arqueamento ou camber, dado também em porcentagem de corda, é a medida de curvatura do perfil sendo a distância da linha de arqueamento média (média das distâncias de um ponto do extradorso ao intradorso) a linha da corda.

Os perfis analisados foram os NACA 4 dígitos pois já estão presentes no banco de dados do software XFLR5 e a nomenclatura facilita a interpretação da mudança da espessura e arqueamento. Na nomenclatura o primeiro dígito refere-se ao arqueamento em relação a corda, o segundo dígito define a posição da máxima curvatura do perfil, o terceiro e quarto dígitos definem a espessura do perfil.

A espessura e arqueamento são as características principais de um perfil aerodinâmico, dessa forma nesse trabalho estudou-se o efeito da espessura mantendo-se os arqueamentos e a posições do arqueamento máximo constantes, variando a espessura, utilizando os seguintes perfis: NACA 2408, NACA 2410, NACA 2412, NACA 2418, NACA 2424.

Para conhecer os efeitos do arqueamento, realizou-se uma simulação com variação do arqueamento mantendo-se constante a espessura e posição do arqueamento máximo, para os seguintes perfis: NACA 0012, NACA 1412, NACA 2412, NACA 4412. Para cada caso, destaca-se como resultados os gráficos dos coeficientes de sustentação e arrasto em função do ângulo de ataque $(\alpha)$, sendo esse ângulo correspondente a corda do perfil em relação a direção do escoamento.

Esses procedimentos já foram realizados em um túnel de vento da própria NACA (National Advisory Committee for Aeronautics), com parâmetros de testes diferentes, e os resultados foram apresentados por Abbot e Doenhoff (1951). Os autores destacam os resultados em algumas variáveis como o máximo coeficiente de sustentação $\left(C_{L m a ́ x}\right)$, a derivada da curva de sustentação $\left(C_{L \alpha}\right)$, o coeficiente de sustentação para ângulo de ataque igual a zero $\left(C_{\llcorner O}\right)$ e o coeficiente de arrasto para ângulo de ataque igual a zero $\left(C_{D O}\right)$ mostrados na curva típica do coeficiente de sustentação pelo ângulo de ataque da Figura 2. 


\section{XIX \\ CENEMI}

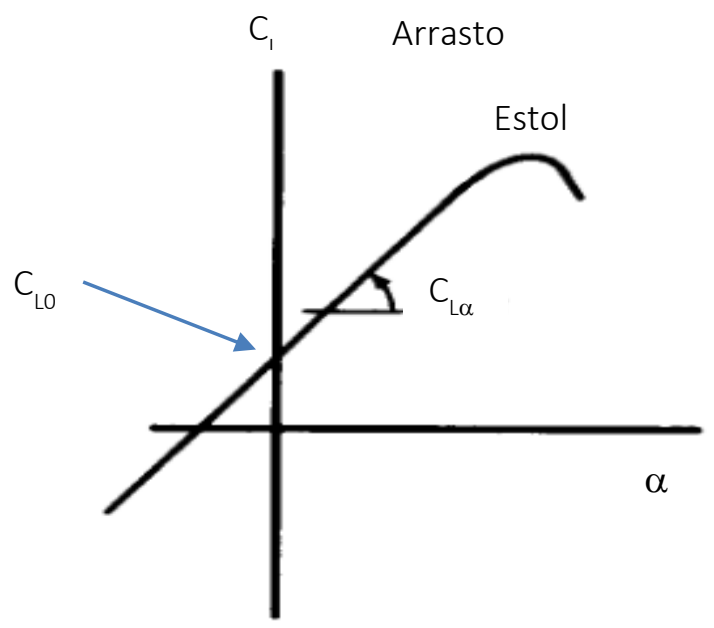

FIGURA 2. Gráfico do coeficiente de sustentação pelo ângulo de ataque. Fonte: Raymer (1992).

Observa-se na Figura 2 pontos importantes para análise dos perfis, o primeiro é o coeficiente de sustentação ou de arrasto para um ângulo de ataque igual a zero, conhecidos como $C_{L O}$ e $C_{D O}$, respectivamente. Esses coeficientes representam o valor da sustentação e arrasto para um voo nivelado, com ressalva dos efeitos que ocorrem na asa e a configuração de asa que modificam esses valores. Especificamente para o gráfico do coeficiente de sustentação, outro valor de importância para a análise é a inclinação da parte linear da curva, que como mostrado na Figura 2, definida como a derivada da curva $\left(C_{L \alpha}\right)$. Esse valor mostra o quanto de sustentação é gerada para uma dada variação do ângulo de ataque, e ele afeta outro ponto de importância na análise, que é conhecido como estol.

O estol é um ponto onde ocorre o maior valor do coeficiente de sustentação ( $\left.C_{L m a ́ x}\right)$ para um dado ângulos de ataque $(\alpha)$, e que para maiores ângulos de ataque, o coeficiente de sustentação cai abruptamente (dependendo do perfil) e o coeficiente de arrasto aumenta rapidamente. Raymer (1992) explica o fenômeno como sendo a perda da capacidade do escoamento de ar se manter "colado" na superfície, gerando vórtices de recirculação de ar e um escoamento turbulento.

A determinação desses coeficientes é realizada através das Equações 1 e 2, para o Coeficiente de sustentação e Coeficiente de arrasto.

$$
\begin{aligned}
& C_{L}=\frac{L}{\text { q.C }} \\
& C_{D}=\frac{D}{\text { q.C }}
\end{aligned}
$$

Onde:

$C_{L}$ - Coeficiente de sustentação (Adimensional); 


\section{XIX \\ CONEMI}

$C_{D}$ - Coeficiente de arrasto (Adimensional);

$\mathrm{L}$ - Força de sustentação $(\mathrm{N} / \mathrm{m})$;

$\mathrm{D}$ - Força de arrasto $(\mathrm{N} / \mathrm{m})$;

$c$ - corda do perfil (m);

q- pressão dinâmica $(\mathrm{Pa})$.

A escolha do perfil reflete nos coeficientes de sustentação e arrasto de uma asa, sendo que o coeficiente de sustentação da asa é menor que a do perfil e o coeficiente de arrasto maior, isso porque ocorre um fenômeno conhecido como vórtice de ponta de asa. Esse fenômeno induz um ângulo de ataque $(\alpha)$ menor nas pontas da asa, e esse efeito decai conforme se afasta da ponta. Logo, maiores valores de $C_{L m a ́ x}$ e $C_{\llcorner o}$ de um perfil refletem em maiores valores dos mesmos para a asa.

Esses coeficientes da asa afetam o desempenho do aeromodelo, como a tração requerida $\left(T_{r}\right)$ para manter o aeromodelo em voo, a menor velocidade para manter um voo nivelado (ou velocidade de estol) (Vestol), a distância necessária para decolagem (SLo e a distância necessária para o pouso (SL), apresentadas nas equações 3, 4, 5 e 6, respectivamente, conforme Rodrigues (2011).

$$
\begin{gathered}
T_{r}=\frac{W}{\frac{C_{L}}{C_{D}}} \\
v_{\text {estol }}=\sqrt{\frac{2 \cdot W}{\rho S C_{L m a ́ x}}} \\
S_{L o}=\frac{1,44 W^{2}}{g \rho S C_{L m a ́ x .}\{T-[D+\mu(W-L)]\}} \\
S_{L}=\frac{1,69 \cdot W^{2}}{g \rho S C_{L m a ́ x}[D+\mu(W-L)]}
\end{gathered}
$$

Onde:

$\mathrm{T}_{\mathrm{r}}$ - Tração requerida para manter a aeronave em voo nivelado $(\mathrm{N})$;

$\mathrm{W}$ - Peso do aeromodelo em voo $(\mathrm{N})$;

Vestol - velocidade de estol $(\mathrm{m} / \mathrm{s})$;

$\rho$ - Massa específica do ar $\left(\mathrm{kg} / \mathrm{m}^{3}\right)$;

$S$ - Área da asa $\left(\mathrm{m}^{2}\right)$;

SLo - Distância necessária para decolagem (m); 
g - Aceleração da gravidade $\left(\mathrm{m} / \mathrm{s}^{2}\right)$;

$\mathrm{T}$ - Tração disponível (N);

$\mu$ - Coeficiente de atrito da roda do trem de pouso com o solo (adimensional);

$\mathrm{S}_{\mathrm{L}}$ - Distância necessária para pouso $(\mathrm{m})$.

Todos os coeficientes presentes nas equações são referentes a um ponto específico da asa conhecido como corda média aerodinâmica. Observa-se das Equações 3, 4, 5 e 6 que um aumento do coeficiente máximo de sustentação diminui a velocidade de estol ( $V_{\text {estol}}$ ), diminui a distância necessária para decolagem $\left(\mathrm{S}_{\mathrm{L}}\right)$ e a distância necessária para pouso $\left(\mathrm{S}_{\mathrm{L}}\right)$. Percebe-se que um aumento da força de arrasto na Equação 6 diminui a distância necessária para pouso, logo um aumento de arrasto pode ser favorável para o pouso.

Considerando que durante a decolagem e o pouso a tração disponível (T) seja constante e que o ângulo de ataque seja igual a zero, os valores da força de sustentação $(L)$ e da força de arrasto (D) dependem diretamente de $C_{L O}$ e $C_{D o}$ respectivamente. Logo um valor de $C_{L O}$ e $C_{D O}$ maior do perfil diminui as distâncias necessária para pouso e decolagem.

Obtendo-se o efeito da espessura e arqueamento nos coeficientes de sustentação e arrasto para perfis em baixos números de Reynolds, utilizados em competições SAE aerodesign, projetou-se um novo perfil com base nos perfis NACA. Os resultados foram comparados ao perfil Eppler 423, pois é um dos principais aerofólios utilizados pelas equipes.

\section{RESULTADOS E DISCUSSÕES}

Para a primeira simulação referente aos efeitos da espessura nos coeficientes de sustentação e arrasto, foram obtidas as curvas das Figura 3 e 4. 


\section{XIX \\ CENEMI}

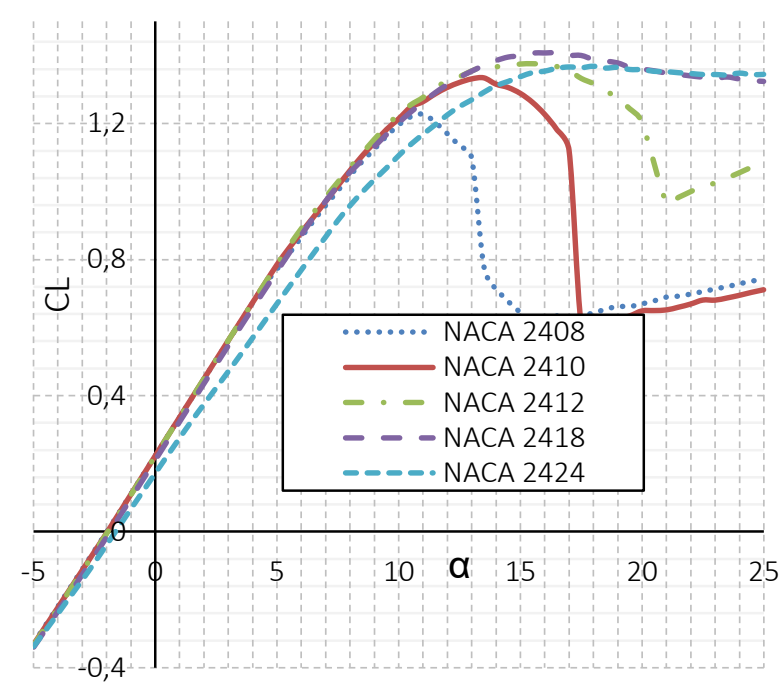

FIGURA 3. Curva coeficiente de sustentação pelo ângulo de ataque para variação de espessura. Fonte: Autor (2019).

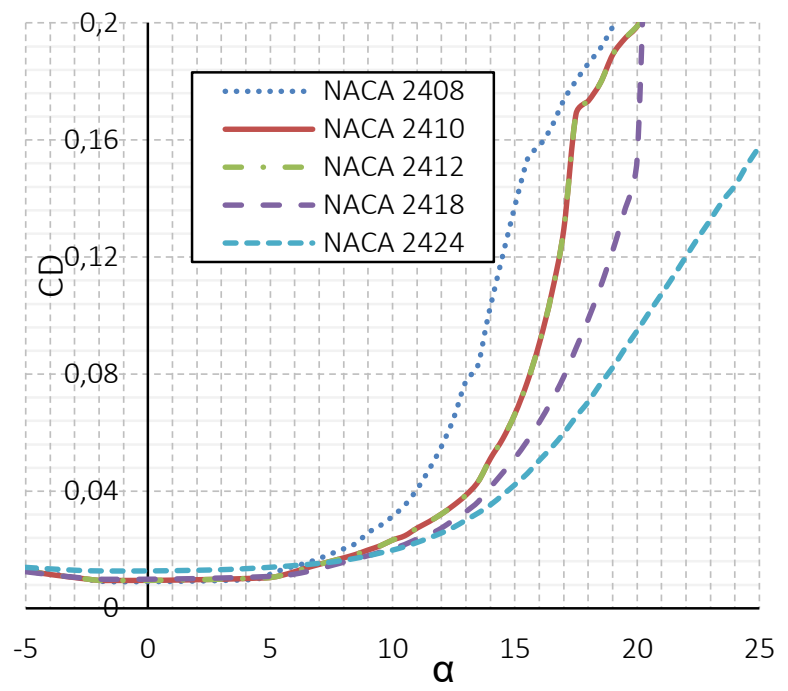

FIGURA 4. Curva coeficiente de arrasto pelo ângulo de ataque para variação de espessura. Fonte: Autor (2019).

A Figura 3 mostra que a mudança da espessura afeta principalmente o coeficiente de sustentação máximo $\left(C_{L m a ́ x}\right)$, de maneira que aumenta conforme a espessura aumenta, até um valor de espessura de $18 \%$ da corda, ou seja, o perfil NACA 2418, sendo que a partir desse valor há decréscimo, com o valor do perfil 2412 sendo próximo do 2424. Além disso, o aumento da espessura diminui significativamente o valor da derivada da curva $\left(C_{L \alpha}\right)$ para espessuras maiores que $18 \%$, como no caso do perfil 2424, onde percebe-se que sua curva tem uma taxa de subida menor que as demais curvas. O valor do coeficiente de sustentação para $\alpha$ igual a zero $\left(C_{L O}\right)$ foi pouco afetado pela mudança da espessura.

Na Figura 4 o aumento da espessura diminui o $C_{D}$ para um mesmo valor do $\alpha$, para valores maiores que $10^{\circ}$, porque diminui-se a inclinação da curva que está associada ao estol. Porém, observa-se que para maiores espessuras o valor do coeficiente de arrasto para ângulo de ataque igual a zero $\left(\mathrm{C}_{\mathrm{DO}}\right)$ tem um leve aumento.

Outra característica importante que foi afetada pela mudança da espessura foi o estol. Para perfis mais finos percebe-se que a perda de sustentação e o arrasto gerado é mais abrupto que para perfis grossos, que apresentam estol mais suave, observado na baixa taxa de declínio da curva. Além disso, nos perfis finos o estol acontece para ângulos de ataques menores comparados aos de maior espessura. 


\section{XIX \\ CONEMI}

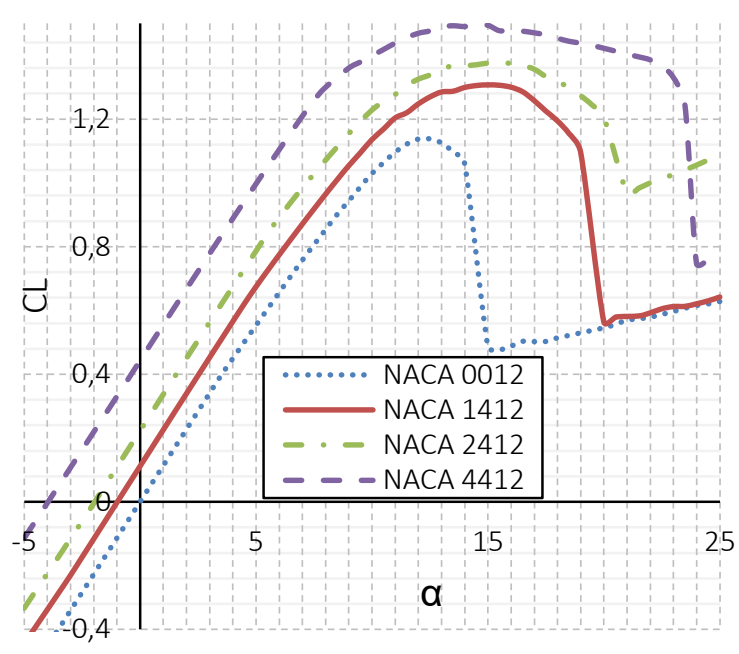

FIGURA 5. Curva coeficiente de sustentação pelo ângulo de ataque para variação de arqueamento. Fonte: Autor (2019).

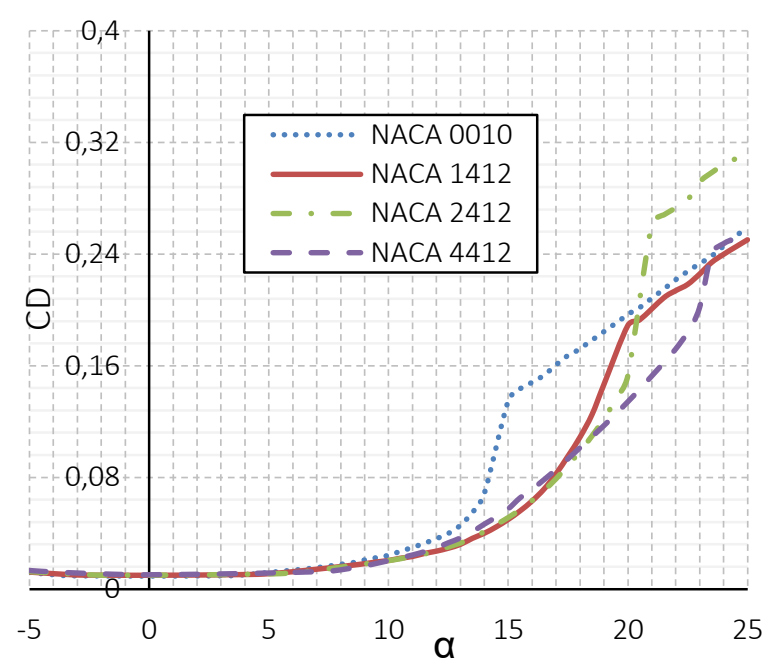

FIGURA 6. Curva coeficiente de arrasto pelo ângulo de ataque para variação de arqueamento. Fonte: Autor (2019).

Para Figura 5 o efeito do arqueamento na sustentação, observa-se que o acréscimo do arqueamento, primeiro dígito da nomenclatura dos perfis (X412), aumenta-se o $C_{\text {Lo }}$ e também o $C_{\text {Lmáx, }}$ mantendo-se igual o valor de $C_{L \alpha}$ para todos os perfis. Para o coeficiente de arrasto na Figura 5, o $C_{D 0}$ permaneceu constante com o aumento do arqueamento e o coeficiente de arrasto para um dado ângulo de ataque depende em qual faixa se está analisando, com o perfil 0010 gerando mais arrasto do que os demais para a menor que $20^{\circ}$, e o perfil 2412 gerando maior arrasto para $\alpha$ maior que $20^{\circ}$. Para o estol, observa-se que para maiores arqueamentos, o ângulo em que ocorre aumenta.

De acordo das análises realizadas, é possível projetar um perfil com a base dos aerofólios NACA 4 dígitos com um grande aumento no arqueamento, maior que $4 \%$ da corda, e com uma espessura intermediária, entre $12 \%$ a $14 \%$ da corda. É possível comparar esse novo perfil com o Eppler 423 de alta sustentação, geralmente utilizados pelas equipes de Aerodesign.

O novo perfil projetado apresenta como base o perfil NACA 4412, com aumento do arqueamento para $12 \%$, mantendo-se as demais características iguais. Logo, considerando a padronização da nomenclatura dos perfis NACAs 4 dígitos, o novo perfil seria denominado de 12412 com $12 \%$ de arqueamento máximo localizado a $40 \%$ da corda e com $12 \%$ de espessura localizado a $30 \%$ da corda. O perfil Eppler 423 tem 10\% de arqueamento máximo localizado a 44\% da corda com $12.5 \%$ de espessura localizado a $24 \%$ da corda (m-selig.ae.illinois.edu/ads/coord_database.html). A Figura 7 mostra uma comparação do novo perfil proposto, o 12412 (em vermelho), com o Eppler 423 (em azul). 


\section{$\mathbf{X I X}$ \\ CENEMI}

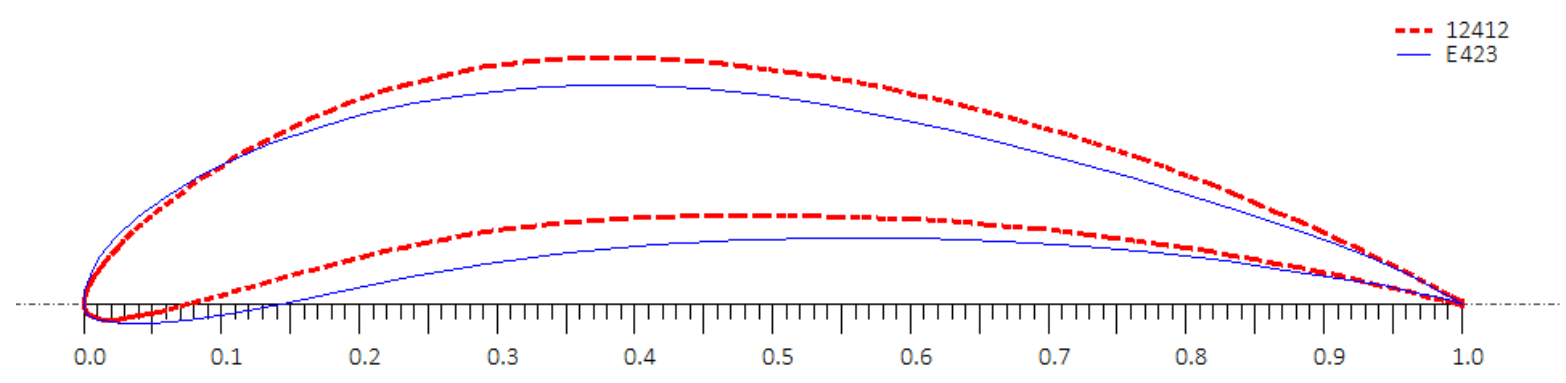

FIGURA 7. Proposta de um perfil 12412 e o perfil Eppler 423. Fonte: Autor (2019).

Os resultados da comparação dos coeficientes em função do ângulo de ataque dos dois perfis são mostrados a seguir.

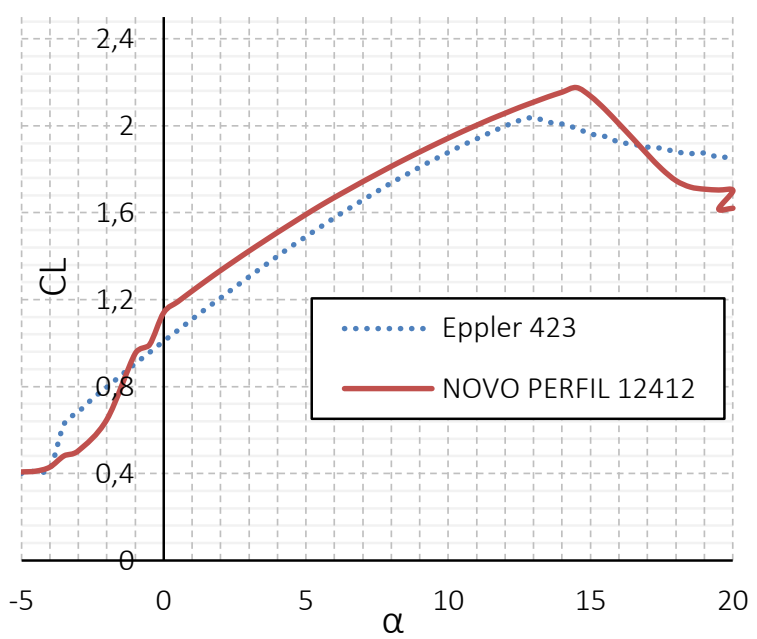

FIGURA 8. Coeficiente de sustentação em função do ângulo de ataque do novo perfil. Fonte: Autor (2019).

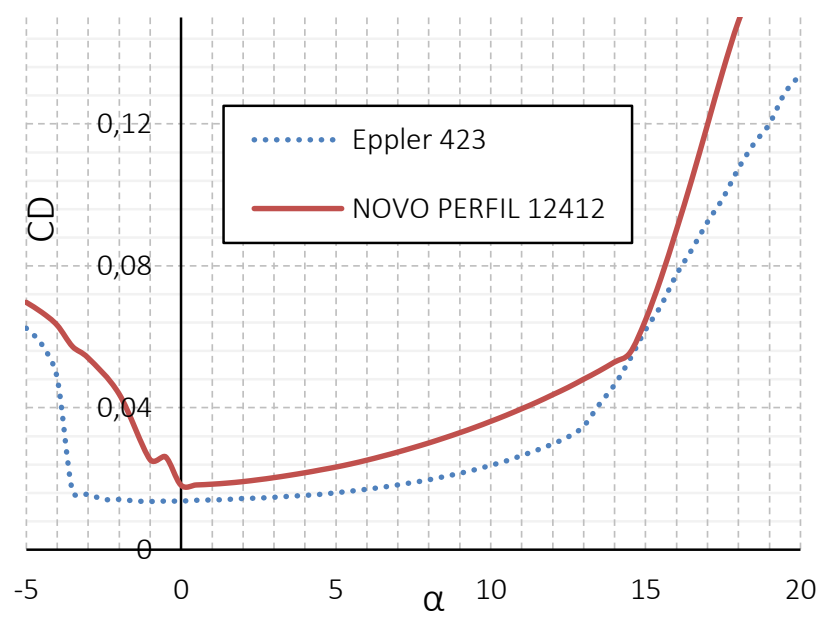

FIGURA 9. Coeficiente de arrasto em função do ângulo de ataque do novo perfil. Fonte: Autor (2019). 


\section{XIX \\ CONEMI}

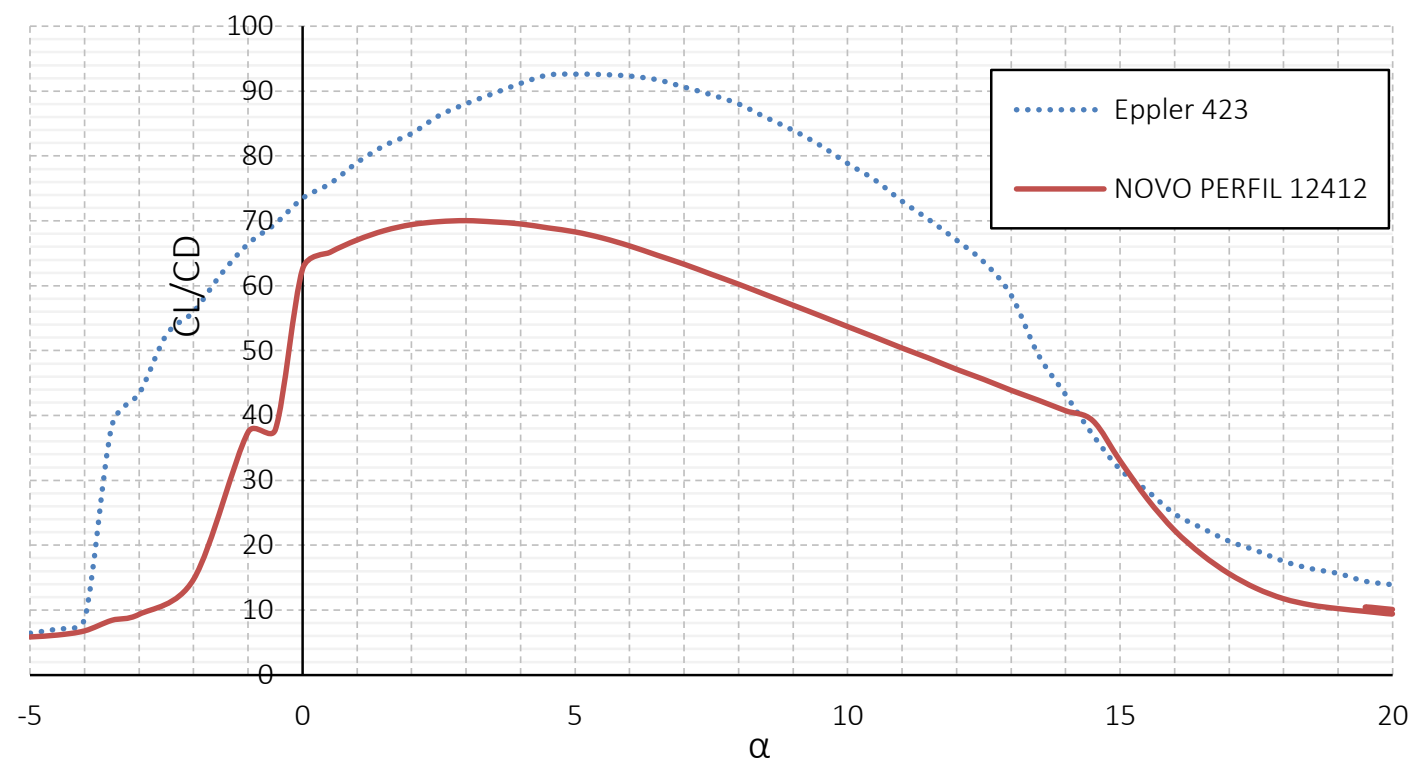

FIGURA 10. Razão CL/CD em função do ângulo de ataque do novo perfil proposto em comparação ao do Eppler 423. Fonte: Autor (2019).

Percebe-se da Figura 8 que o $C_{\text {Lmáx }}$ e o $C_{L 0}$ do novo perfil proposto é maior que do perfil Eppler 423, e na Figura 9 o arrasto total e o C $C_{D O}$ do novo perfil é maior. Com o aumento dessas variáveis, e analisando em conjunto com a Equações 4, 5 e 6 o novo perfil consegue melhores característica com

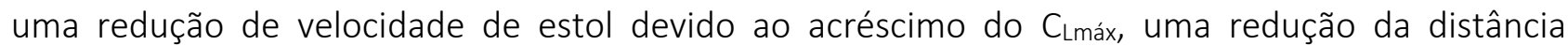
necessária para a decolagem e para o pouso devido ao aumento $C_{\text {Lmáx, }}$ do $C_{L O}$ e do aumento do $C_{D O}$.

Porém, conforme mostra a Figura 10, a razão CL/CD ou a eficiência aerodinâmica do novo perfil é menor que a do Eppler 423, e analisando conjuntamente com a Equação 3, resulta em uma maior tração requerida para se manter um voo nivelado do aeromodelo em comparação com o perfil Eppler 423.

Como no regulamento do torneio de acesso 2019 a única restrição ao projeto foi a envergadura da asa, a equipe Tuiuiú optou pelo perfil Eppler 423 pois tem maior facilidade de construção devido ao menor arqueamento. Porém, pensando na competição de 2020, o novo perfil 12412 pode ser utilizado, pois nas competições há restrição do comprimento de pista necessária para pouso e decolagem, e como demostrado, o novo perfil proposto obteve melhor resultado nessas características.

\section{CONCLUSÕES}

Esse trabalho apresentou o efeito da espessura e arqueamento (ou camber) nos coeficientes de sustentação e arrasto, através das análises dos perfis NACAs 4 dígitos no software XFLR5. 


\section{$\mathbf{X I X}$ \\ CENEMI}

Constatou-se com as análises dos perfis NACAs 4 dígitos que com o aumento da espessura o arrasto total e o $C_{D o}$ aumentou. Com o aumento do arqueamento, aumentou-se o $C_{L m a ́ x}$ e o $C_{L O}$, enquanto o arrasto total foi menor para perfis mais arqueados.

Posteriormente a análise dos efeitos das características geométricas, criou-se um perfil específico para o projeto do aeromodelo com $12 \%$ de arqueamento máximo localizado a $40 \%$ da corda e com 12 \% de espessura, o perfil 12412. Comparou-se o novo perfil proposto com o aerofólio Eppler 423, e obteve-se melhores características de desempenho como a velocidade de estol e a

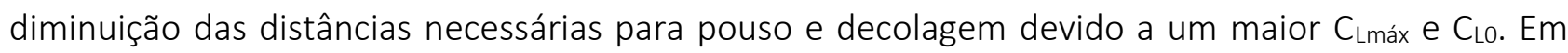
contrapartida, houve um aumento na tração requerida do motor para manter o aeromodelo em voo, devido a um aumento de $C_{D}$ e $C_{D o}$ e decréscimo da eficiência aerodinâmica.

Logo, a criação e desenvolvimento de novos perfis se faz necessário pois cada ano novos requisitos são lançados nas competições do aerodesign, e os perfis disponíveis podem apresentar uma resposta que não seja satisfatória, necessitando utilizar os conhecimentos dos efeitos das características geométrica para criação de um aerofólio que se adapte ao projeto da equipe e que cumpra os requisitos.

\section{REFERÊNCIAS}

ABBOTT, I. H.; DOENHOFF, A. E. V. Theory of Wing Sections. Dover Publications, New York, EUA, 693 p., 1959.

ANDERSON, J. D. Fundamentals of Aerodynamics. McGraw-Hill, New York, EUA, 892 p., 2001.

MATHIAS, M. S. Projeto aerodinâmico de aerofólios e asa para um avião da classe commuter. São Carlos. Trabalho de Conclusão de Curso, 80 p., 2015

RAYMER, D.P. Aircraft Design: A Conceptual Approach. American Institute of Aeronautics and Astronautics, Washington, EUA, 745 p., 1992.

RODRIGUES, L. E. M. J. Fundamentos da engenharia aeronáutica: aplicações ao projeto SAE aerodesign. Edição do autor, São Paulo, Brasil, 544 p., 2011. 